\title{
Sinonasal natural killer/T-cell lymphoma presenting as pyrexia of unknown origin with nasal symptoms
}

\author{
Betsy KH Soon ${ }^{1}$, MBBS, Xin-Rong $\underline{\operatorname{Lim}}^{1}$, MBBS, Deborah $H L \underline{N g}^{1}$, MRCP, MBChB, Ming-Yann $\underline{\operatorname{Lim}}^{2}$, MRCSG, DOHNS
}

\begin{abstract}
A 68-year-old Chinese man presented with an eight-month history of pyrexia of unknown origin and chronic sinusitis despite multiple courses of antibiotics. He underwent extensive investigations, including workups for infections, chronic granulomatous diseases and malignancy. Nasal biopsies were performed twice under local anaesthesia, but did not show any evidence of malignancy. Eventually, the patient was diagnosed with natural killer (NK)/T-cell lymphoma, nasal variant, based on histopathological findings from harvested deep tissue obtained via functional endoscopic sinus surgery. This study highlights that, for patients presenting with pyrexia of unknown origin and nasal symptoms, NK/T-cell lymphoma must be considered as a differential diagnosis. Generous amounts of tissue should be harvested under general anaesthesia rather than limited tissue under local anaesthesia, in order to facilitate and ensure a definitive diagnosis.
\end{abstract}

Keywords: lymphoma, natural killer/T-cell, pyrexia of unknown origin, sinus

\section{INTRODUCTION}

Sinonasal lymphoma is a rare condition that can be difficult to diagnose. This diagnostic difficulty stems primarily from physicians having a low index of suspicion for a relatively uncommon condition. This is coupled with the need for a judicious choice of biopsy site to obtain viable tissue that would enable histological diagnosis. To achieve this is difficult, as the nose is often full of slough and necrotic tissue. Also, biopsy under local anaesthesia may preclude optimal examination, and hence produce inconclusive histology.

Sinonasal lymphoma is associated with poor prognosis. ${ }^{(1)}$ The disease is classified into four stages according to the Ann Arbor classification. ${ }^{(2)}$ In a recent local case series, 21 patients with stage I-IV disease (stage I, $\mathrm{n}=13$; stage II, $\mathrm{n}=5$; stage III, $\mathrm{n}=0$; stage IV, $\mathrm{n}=3$ ) were treated with radiotherapy, in addition to 16 patients who received chemotherapy. ${ }^{(1)}$ The median age of patients was 44 (range 27-86) years in the radiotherapy group. The two-year overall survival rate was only $52.8 \%$. Among the patients treated with radiotherapy, five patients had rapidly progressive disease, with a median survival of 89 days from diagnosis. Of the remaining 16 patients who had complete remission, four patients relapsed during the follow-up period (median follow-up, 23.4 months).

Herein, we report the case of a patient with fever and chronic sinusitis refractory to medical treatment, in whom the diagnosis of natural killer (NK)/T-cell lymphoma, nasal variant, was confirmed only upon histopathology of harvested deep tissue. This case illustrates the diagnostic challenges associated with sinonasal lymphoma.

\section{CASE REPORT}

A 68-year-old Chinese man with a known history of chronic sinusitis was admitted in December 2011 for investigation of pyrexia of unknown origin with chronic nasal discharge.
In the past few years, the patient had sought medical attention at several different hospitals for complaints of intermittent mucopurulent right nasal discharge. Examination on each occasion had revealed congestion of the right nasal mucosa and fullness of the right paranasal sinuses (PNS). Multiple biopsies of the postnasal space had been negative for malignancy. He had been treated with nasal douche, regular nasal toilets and multiple courses of antibiotics. His nasal symptoms, which seemed to show slight improvement with oral steroids, had recurred upon stopping medication.

Computed tomography (CT) of the sinuses performed in May 2011 showed total opacification of the right maxillary sinus, ethmoid sinus, frontal sinus, right nasal cavity and nasopharynx. He also underwent CT of the abdomen and pelvis, and endoscopy, which did not reveal any evidence of malignancy. Chest radiography was unremarkable.

The patient was admitted in June 2011 with similar symptoms of intermittent fever, right blocked nose and right mucopurulent nasal discharge. Nasal endoscopy at the time had revealed an oedematous right anterior nasal cavity with mucopus and normal left anterior nasal cavity. There was also evidence of polypoid tissue at the posterior of the nasal cavity. Biopsy of the lesion had shown mucoid material mixed with fibrinopurulent exudate, but no evidence of malignancy. Fungal stains did not reveal any definite fungal elements. As the nasal swab grew methicillinsensitive Staphylococcus aureus, the patient was treated with a course of piperacillin-tazobactam followed by co-amoxiclav. Repeat nasal biopsy was conducted in view of the persistent fibrinous exudates covering the polypoid mucosa in the right PNS, and again, histological features were suggestive of benign inflammatory exudates.

The patient, who continued to be followed up at outpatient clinics, received multiple courses of antibiotics, completing a total of more than three months of oral antimicrobial treatment. 

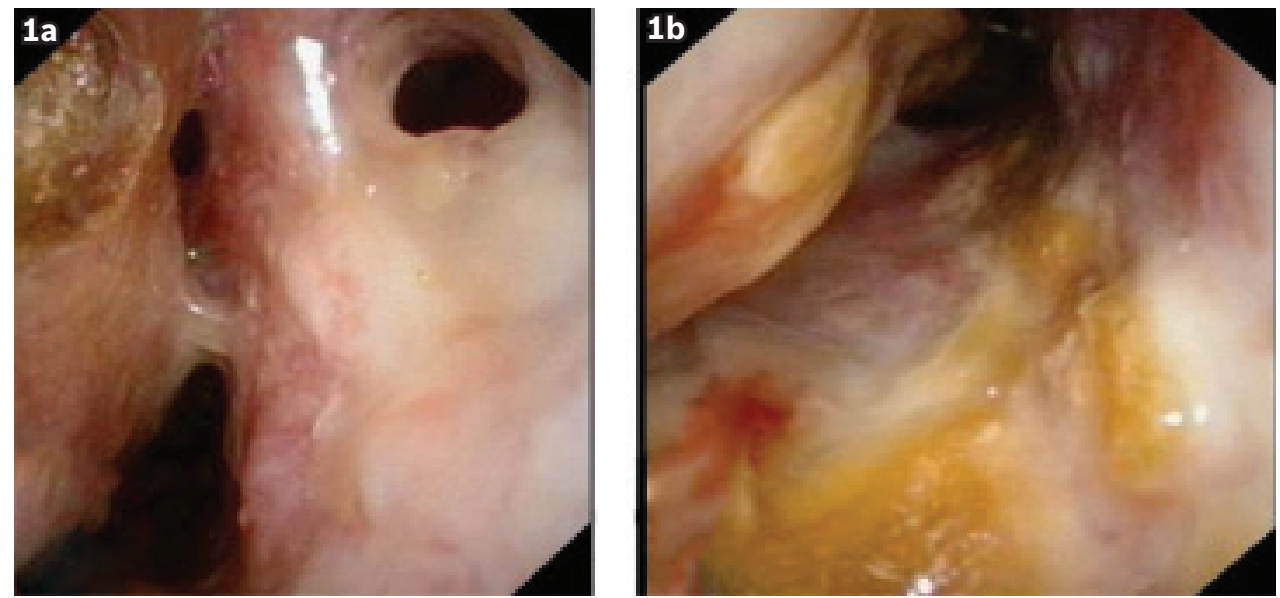

Fig. 1 Nasal endoscopic images from October 2011 show (a) nasal septal perforation and (b) bilateral crusting of the anterior nasal cavities
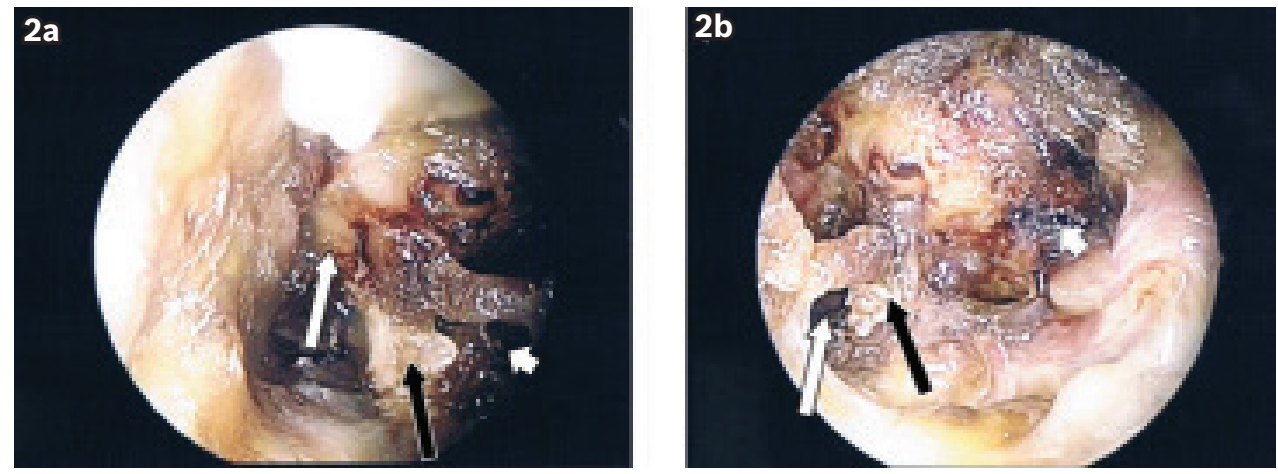

Fig. 2 Photographs taken during functional endoscopic sinus surgery show (a) the right nasal cavity with crusting (white arrow), exposed septal cartilage (black arrow) and septal perforation (arrowhead); and (b) the left nasal cavity with septal perforation (white arrow), exposed septal cartilage (black arrow) and crusting (arrowhead).

He was also seen at the outpatient infectious disease clinic for latent syphilis in November 2011, and was prescribed four weeks of oral doxycycline for empirical treatment of late latent syphilis. In view of his persistent symptoms, repeat otolaryngological endoscopy was performed in October 2011, which revealed nasal septal perforation and bilateral crusting of his anterior nasal cavities (Fig. 1).

In December 2011, the patient again presented with persistent fever and nasal congestion. Routine haematological and biochemical investigations showed leucocytosis (white blood cell count, $\left.13.7 \times 10^{9} / \mathrm{L}\right)$ with neutrophilia $(79.6 \%)$, mild normocytic anaemia (haemoglobin, $11.4 \mathrm{~g} / \mathrm{dL}$ ), raised C-reactive protein $(66.1 \mathrm{mg} / \mathrm{L})$ and procalcitonin $(0.08 \mathrm{ug} / \mathrm{L})$. Blood cultures and nasal swab bacterial and fungal cultures were negative, as were sputum acid-fast bacilli smears and cultures for mycobacteria. Human immunodeficiency virus screening test was also negative. Further investigations for workup for chronic granulomatous disease, including nasal acid-fast bacilli smears and cultures, and antineutrophil cytoplasmic antibody (ANCA) test, did not yield any significant results. CT of the thorax, abdomen and pelvis also did not reveal any abnormalities. However, CT of the sinuses showed polypoidal soft tissue thickening in the left nasal cavity extending into the choana and pansinusitis, suggestive of sinusitis. Nasal examination revealed extensive slough and crusting of the nasal mucosa of the septum, inferior turbinates and lateral wall and floor of the nose. Nasal toilet was almost impossible to perform, as the crusts were totally adherent to the mucosa and the patient was in severe pain despite attempts to moisten the crusts before removal.

In view of the patient's persistent fever and nasal symptoms, functional endoscopic sinus surgery (FESS) was scheduled with an aim to debride necrotic sloughy tissue, establish ventilation of the sinuses and obtain deep nasal tissue for biopsy. During FESS, anterior nasal septum perforation with exposed cartilage was seen. The mucosa of the nasal floor, lateral nasal wall and both inferior turbinates were found to be sloughy and necrotic (Fig. 2). The patient underwent bilateral middle meatal antrostomies, ethmoidectomies, sphenoidotomies and frontal sinusotomies. Extensive debridement of the necrotic mucosa was performed, and tissue was sent for histopathological analysis. Both the inferior turbinates were also removed and sent for histology. The patient made a good postoperative recovery, but had repeated nasal crusting after the surgery, which required regular nasal douching and nasal toilet.

The final histological features were consistent with extranodal NK/T-cell lymphoma, nasal variant. Positron emission tomography showed increased fluorodeoxyglucose avidity in the anterior nasal region bilaterally and the remnant bony nasal septum posteriorly, suggestive of residual metabolically active foci of lymphoma. The NK/T-cell lymphoma was classified as 
stage I disease according to the Ann Arbor classification. Bone marrow aspiration showed mild hypercellular marrow, with no abnormal lymphoid infiltrate. Following further discussion, the patient underwent radiotherapy and chemotherapy for the treatment of lymphoma. He subsequently developed a palatal fistula during radiotherapy, which was managed using nasogastric tube feeding.

\section{DISCUSSION}

Sinonasal NK/T-cell lymphoma is known to be one of the most aggressive lymphomas, causing progressive midline destruction of the nasal or mid-facial bones. ${ }^{(3)}$ Although generally rare, sinonasal NK/T-cell lymphoma accounts for $2.6 \%-6.7 \%$ of all lymphomas, but less than $0.5 \%$ of lymphoma cases in Western populations. ${ }^{(4)}$ In Asians, after the gastrointestinal tract, the sinonasal tract is the next most common site of extranodal lymphoma. ${ }^{(5,6)}$

Common presenting symptoms include nasal obstruction, rhinorrhoea, epistaxis, and although rarely, facial swelling and orbital symptoms such as ptosis or limitation of eye movement. ${ }^{(5)}$ Examination findings often reveal nasal discharge, enlarged turbinates or a deviated nasal septum. Our patient had chronic rhinosinusitis symptoms refractory to multiple courses of antibiotics, and during the later course of the disease, anterior nasal septum perforation in addition to 'B' symptoms of fever and weight loss. Other studies have reported destructive lesions such as perforating palatal ulcer and nasal septal perforation in patients with nasal NK/T-cell lymphoma.(7)

The present case report highlights two important points. Firstly, NK/T-cell lymphoma should be considered in patients with refractory sinusitis. Secondly, comprehensive biopsies under general anaesthesia are required to establish definitive diagnosis and should be preferred over limited biopsies under local anaesthesia due to the confounding presence of extensive necrotic tissue.

In our patient, the symptoms of fever and chronic sinusitis refractory to medical treatment were present for at least eight months before the diagnosis was made. This illustrates the challenge involved in making a diagnosis of nasal NK/T-cell lymphoma, as its signs and symptoms often mimic that of chronic rhinosinusitis. ${ }^{(6)}$ Sands et al observed in a series of four patients that extranodal nasal NK/T-cell lymphoma being mistaken for chronic sinusitis resulted in therapeutic delays in these patients ( mean time to diagnosis $=21.5$ months) ${ }^{\left({ }^{(8)}\right.}$

The initial nasal biopsies from our patient revealed fibrin and acute inflammatory exudates. Possible explanations for obtaining such nonspecific inflammatory exudates during biopsies may be: (a) the coexistence of lymphoma with secondary rhinosinusitis; and (b) necrosis and inflammation resulting from the destruction of adjacent anatomic structures caused by the NK/T-cell lymphoma.

Previous reports suggest that superficial biopsies may be inadequate for deep-seated lesions such as lymphoma, which can present as submucosal lesions rather than ulcerative or polypoid lesions. ${ }^{(9)}$ In a similar fashion, a definitive diagnosis of NK/T-cell lymphoma was only made in our patient after deep-seated histological specimens were obtained during FESS. This clearly shows that simple punch biopsies may not harvest adequate amounts of tissue in these patients. Indeed, surgical procedures such as FESS, turbinectomy or the Caldwell-Luc procedure have been reported to be more useful for harvesting adequate biopsy specimens for the pathological examination of sinonasal lymphoma. ${ }^{(9)}$

In patients with persistent symptoms of sinusitis despite 'adequate' treatment, a high index of suspicion should be exercised for more sinister conditions such as nasal lymphoma. Apart from chronic sinusitis symptoms refractory to conventional treatment, our patient also had the red-flag 'B' symptoms of fever and weight loss. Generally, the prognosis for extranodal NK/T-cell lymphoma is poor despite localised disease at presentation. However, the prognosis is said to improve if the diagnosis is made earlier. ${ }^{(10)}$ Therefore, early diagnosis and treatment of sinonasal lymphoma is essential especially in patients of Asian origin, for whom the prognosis and outcomes have been reported to be more dismal compared to Western populations. ${ }^{(11,12)}$

\section{ACKNOWLEDGEMENTS}

The authors would like to thank Dr Oon-Tek Ng, Consultant at the Department of Infectious Disease, Tan Tock Seng Hospital (TTSH) and Dr Julian CY Lee, Consultant at the Department of Otorhinolaryngology, TTSH for their valuable input and guidance.

\section{REFERENCES}

1. Tham IW, Lee KM, Yap SP, Loong SL. Outcome of patients with nasal natural killer (NK)/T-cell lymphoma treated with radiotherapy, with or without chemotherapy. Head Neck 2006; 28:126-34

2. Armitage JO. Staging non-Hodgkin lymphoma. CA Cancer J Clin 2005; 55:368-76.

3. Parker NP, Pearlman AN, Conley DB, Kern RC, Chandra RK. The dilemma of midline destructive lesions: a case series and diagnostic review. Am J Otolaryngol 2010; 31:104-9.

4. Ho F, Choy D, Loke SL, et al. Polymorphic reticulosis and conventional lymphomas of the nose and upper aerodigestive tract: a clinicopathologic study of 70 cases, and immunophenotypic studies of 16 cases. Hum Pathol 1990; 21:1041-50.

5. Woo JS, Kim JM, Lee SH, et al. Clinical analysis of extranodal nonHodgkin's lymphoma in the sinonasal tract. Eur Arch Otorhinolaryngol 2004; 261:197-201.

6. Kyrmizakis DE, Hajiioannou JK, Koutsopoulos AV, et al. Primary nasal non-Hodgkin lymphomas presented initially as benign disease. Am J Otolaryngol 2006; 27:217-20.

7. Saleh KA, Razif, Gendeh BS, et al. Nasal NK/T cell lymphoma mimicking an iatrogenic lateral nasal wall infection: a diagnostic dilemma. Med J Malaysia 2011; 66:160-1.

8. Sands NB, Tewfik MA, Hwang SY, Desrosiers M. Extranodal T-cell lymphoma of the sinonasal tract presenting as severe rhinitis: case series. J Otolaryngol Head Neck Surg 2008; 37:528-33.

9. Yen TT, Wang RC, Jiang RS, et al. The diagnosis of sinonasal lymphoma: a challenge for rhinologists. Eur Arch Otorhinolaryngol 2012; 269:1463-9.

10. Yok-Lam K. The diagnosis and management of extranodal NK/T-Cell lymphoma, nasal-type and aggressive NK-Cell leukemia. J Clin Exp Hematop 2011; 51:21-8.

11. Cuadra-Garcia I, Proulx GM, Wu CL, et al. Sinonasal lymphoma: a clinicopathologic analysis of 58 cases from the Massachusetts General Hospital. Am J Surg Pathol 1999; 23:1356-69.

12. Liang $R$, Todd D, Chan TK, et al. Nasal lymphoma. A retrospective analysis of 60 cases. Cancer 1990; 66:2205-9. 